\title{
Performance Analysis of the Cache Conscious-Generalized Search Tree
}

\author{
Won-Sik Kim ${ }^{1}$, Woong-Kee Loh $^{2}$, and Wook-Shin Han ${ }^{1, \star}$ \\ ${ }^{1}$ Department of Computer Engineering \\ Kyungpook National University, Korea \\ wskim@www-db.knu.ac.kr, wshan@knu.ac.kr \\ ${ }^{2}$ Department of Computer Science \& \\ Advanced Information Technology Research Center (AITrc) \\ Korea Advanced Institute of Science and Technology (KAIST), Korea \\ woong@mozart.kaist.ac.kr
}

\begin{abstract}
Recently, a main memory index structure called the cache conscious-generalized search tree (CC-GiST) was proposed. The CCGiST is such a novel index structure that it can be used for implementing all the existing cache conscious trees with the minimal efforts. It incorporates the pointer compression and the key compression techniques, which were adopted by the existing cache conscious trees to reduce the cache misses, in a single framework. In this paper, we formally analyze the performance of the CC-GiST. We compare the performance of the CC-GiST with the existing cache conscious trees. The result shows that the CC-GiST has the negligible overhead for supporting all the existing cache conscious trees in a single framework, and the performance of the tree is almost unaffected.
\end{abstract}

Keywords: cache conscious tree, generalized search tree, pointer compression, key compression.

\section{Introduction}

According to the advance of technologies, the speed gap between CPU and main memory is getting larger every year [5, 10. Due to the speed gap, it was perceived important to make the most use of the cache residing between CPU and main memory, and there have been a lot of research efforts on this issue [1, 3, 4, 5, 6, Among those is the research on cache conscious trees for reducing the cost for accessing main memory indexes [2, 8, 10]. Cache conscious trees were designed to cause as few cache misses as possible based on the characteristics of the cache. The most widely known cache conscious trees are the $\mathrm{CSB}^{+}$-tree 10 , the pkBtree [2], and the CR-tree [8].

Recently, we proposed the cache conscious-generalized search tree (CC-GiST) 9, which is an extension of the disk based GiST [7. The CC-GiST can be used for implementing all the existing cache conscious trees with the minimal efforts.

\footnotetext{
^ Corresponding author.
} 
It incorporates the pointer compression and the key compression techniques, which were adopted by the existing cache conscious trees to reduce the cache misses, in a single framework. In this paper, we provide the formal analysis on the performance of the CC-GiST. We compare the performance of the CC-GiST with the existing cache conscious trees. As the result, the CC-GiST has the negligible overhead for supporting all the existing cache conscious trees in a single framework, and the performance of the tree is almost unaffected.

The rest of this paper consists of the following. In Section 2, we briefly explain on the existing cache conscious trees and the CC-GiST. In Section 3, we analyze the performance of the CC-GiST. Finally, we conclude this paper in Section 4.

\section{Related Work}

In this section, we analyze the existing cache conscious trees and derive the pointer compression and the key compression techniques used by the trees. We also briefly explain the CC-GiST 9 .

\subsection{Cache Conscious Trees}

The cache conscious trees use the techniques for reducing the memory size of the indexes and for increasing the blocking factors of the nodes in the indexes. We call the techniques as compressions in this paper. In general, when the blocking factor gets larger, since it is more probable that the related data are contained in the same cacheline, it can reduce the cache misses 1 . We can group the existing cache conscious trees into two categories according to their compression techniques: (1) those based on pointer compression and (2) those based on key compression. The cache conscious trees based on pointer compression remove the subset of pointers to child nodes and thus increase the blocking factors in the internal nodes. The examples are the Cache Sensitive $\mathrm{B}^{+}$-tree $\left(\mathrm{CSB}^{+}\right.$-tree) [10] and the Segmented $\mathrm{CSB}^{+}$-tree [10]. The cache conscious trees based on key compression store the keys of shorter sizes and thus increase the blocking factors in the internal and the leaf nodes. The examples are the Partial Key B-tree (pkB-tree) [2] and the Cache Conscious R-tree (CR-tree) [8].

Figure 1shows an example of the $\mathrm{CSB}^{+}$-tree. All the child nodes of an internal node are stored in a node group, which is represented by a dashed rectangle in the figure, and the internal node manages only one pointer to the first child node in the node group, which is represented by a solid arrow in the figure. Since the child nodes in a node group are stored physically consecutively in main memory, the pointer to any child node in the node group can be easily found even by managing only one pointer. In this manner, the performance of the tree can be enhanced by reducing the number of pointers and thus increasing the blocking factor.

${ }^{1}$ The data in main memory are loaded into cache in the unit of cacheline. The usual size a cacheline is $64 \sim 128$ bytes, and the size of a node in cache conscious trees is the integer multiple of the size of cacheline. 


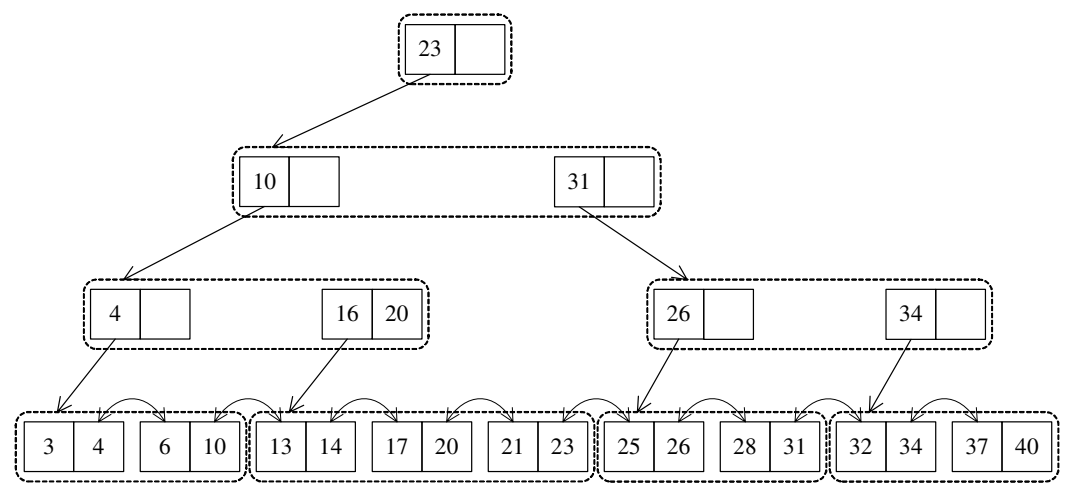

Fig. 1. An Example of the $\mathrm{CSB}^{+}-$Tree

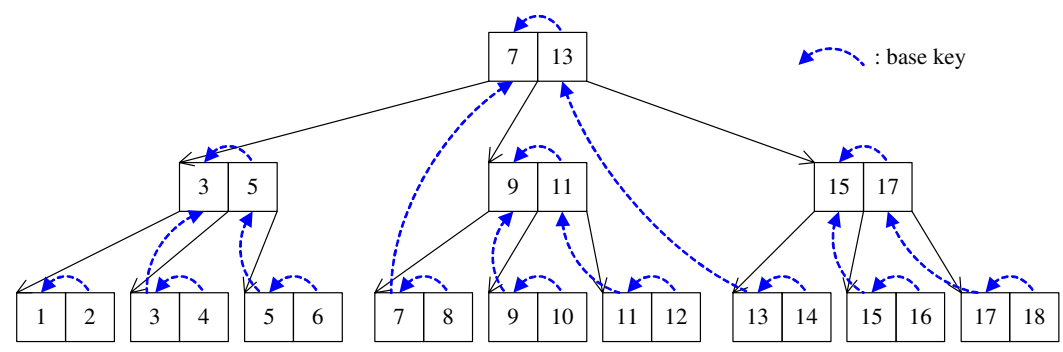

Fig. 2. An Example of the pkB-Tree

The $\mathrm{CSB}^{+}$-tree has a problem of increased update cost. When there happens a split or a merge of nodes while inserting or deleting data in the tree, to maintain the child nodes in a node group stored physically consecutively even after the split or the merge, all the nodes stored in the node group should be copied. The Segmented $\mathrm{CSB}^{+}$-tree is a variant of the $\mathrm{CSB}^{+}$-tree to tackle the problem, and stores the child nodes of an internal node in one or more segments rather than only one node group to reduce the copy cost. Only the child nodes in the same segment are stored physically consecutively, and the internal node manages only the pointers to the first nodes of the segments. The $\mathrm{CSB}^{+}$-tree in Figure 1 can be regarded as a Segmented $\mathrm{CSB}^{+}$-tree that has only one segment in each internal node.

The pkB-tree, which is based on key compression, manages only the different part between adjacent keys in a node [2]. The tree stores only the short part of keys rather than the long whole keys, which results in the increasing of the blocking factor of the node. Figure 2 shows an example of the pkB-tree. The solid arrow represents the pointer to a child node, and the dashed arrow represents the base key that is used for key compression. The pkB-tree performs key compression by managing only the different part between the base key and the key to be compressed. 


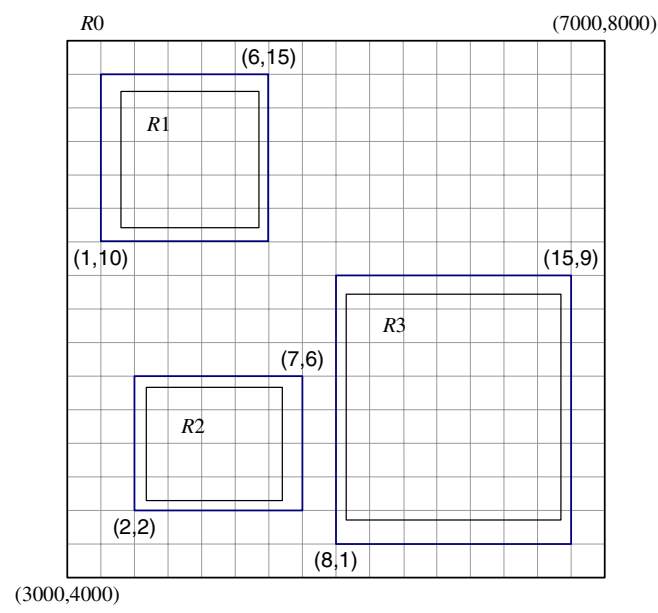

Fig. 3. An Example of QRMBRs in the CR-Tree

The CR-tree, which is also based on key compression, manages the Quantized Relative Representation of MBR (QRMBR) rather than the actual MBR [8]. The QRMBR is generated by quantizing the relative coordinates of the actual MBR to the parent node's MBR. Since the coordinates of the QRMBR have smaller size than those of the actual MBR, the CR-tree stores more entries in a node. Figure 3 shows an example of QRMBRs in the CR-tree. The outermost rectangle $R 0$ is the parent node's MBR, and the inner rectangles $R 1, R 2$, and $R 3$ are child nodes' MBRs. The thick rectangles surrounding the child nodes' MBRs are their QRMBRs generated relatively to $R 0$. Since the relative coordinates are quantized in 16 units, which require only four bits, the space to store the coordinates can be reduced.

\subsection{Cache Conscious-Generalized Search Tree (CC-GiST)}

The CC-GiST is a balanced search tree with the blocking factor between $k M$ and $M$, where $k$ is the minimum fill factor $\left(\frac{2}{M} \leq k \leq \frac{1}{2}\right)$, and $M$ is the maximum blocking factor in the tree. An exception is the root node that has the blocking factor between 2 and $M$.

Based on the pointer compression technique, child nodes are stored in segments, and parent nodes manage only the pointers to the segments in the CCGiST. A segment contains one or more adjacent child nodes stored physically consecutively in main memory. The CC-GiST supports both the fixed- and the variable-size segments. Whether to use the fixed- or the variable-size segment is determined by the flag IsFixedSizeSegment. The CC-GiST manages the variable MaxNumOfNodesInSegment representing the maximum number of child nodes contained in a segment.

An internal node in the CC-GiST manages ListOfSegmentPointers, a list of pointers to the segments of the node. It also manages ListOfNumOfChildNodesInSegment, a list of the numbers of the child nodes in each segment of the node. 
A pointer to a child node of an internal node in the CC-GiST, which is represented as NodePointer $=$ (SegmentPointer, NodeOrderlnSegment $)$, consists of a pointer to the segment containing the child node and the order of the child node in the segment (NodeOrderlnSegment $\geq 0$ ). The NodePointer is used to specify a child node of an internal node, and is not actually stored in the CC-GiST.

Based on the key compression technique, the CC-GiST manages the flag StoreBaseKeylnNode to indicate whether to contain the space to store the base key in a node as in the CR-tree. If the flag is set to TRUE, every node in the CC-GiST contains the space for the base key. The CC-GiST provides an object AncestorKeyStack to store the base keys from the ancestor nodes on the search path used when compressing and decompressing the keys. The AncestorKeyStack is temporarily used while searching, insertion, and deletion, and is not actually stored in the CC-GiST. A key in a node in the CC-GiST is represented by a predicate as in the GiST. A key predicate has one or more free variables and becomes TRUE when the variables are instantiated with the values of any tuple reachable from the pointer associated with the predicate. A node in the CC-GiST manages a list of key predicates.

\section{Performance Analysis}

In this section, we analyze the performance of the CC-GiST [9]. Given the node size, the performance of the cache conscious trees is inversely proportional to the average number of entries in a node. The less the number of entries is, the more probably the cache misses are incurred, which causes the increase of main memory accesses and thus the performance degradation of the cache conscious tress. Therefore, the existing cache conscious trees tried to increase the number of entries in a node using the pointer and the key compression techniques. In this section, we compare the number of entries in an internal node in the cache conscious trees implemented using the CC-GiST with those in the existing cache conscious trees.

Figure 4 shows the structure of an internal node in the CC-GiST. The internal node consists of a header, a base key, a key list, a list of the numbers of child nodes in segments, and a list of segment pointers. The header contains the number of keys $(M)$, the number of segments $(N)$, and a few flags. The base key can be excluded in the node according to the cache conscious tree implemented using the CC-GiST. In the figure, the dashed arrows indicate the directions of inserting new keys and segments.

\begin{tabular}{|c|c|c|c|c|c|}
\hline \multirow{3}{*}{ Header } & $\begin{array}{c}\text { Base } \\
\text { Key }\end{array}$ & Keys $(M)$ & & $\begin{array}{c}\text { \# Nodes in } \\
\text { Scgments }(N)\end{array}$ & $\begin{array}{c}\text { Segment } \\
\text { Pointers }(N)\end{array}$ \\
\hline
\end{tabular}

Fig. 4. Structure of an Internal Node in the CC-GiST 
The number of entries in an internal node in the CC-GiST should be computed according to whether to perform the pointer compression or not. When the pointer compression is performed $(M>N)$, the number of entries is as the following:

$$
\frac{\text { NodeSize }-(\text { HeaderSize }+ \text { BaseKeySize }+ \text { SegNodesListSize }+ \text { SegPtrListSize })}{\text { KeySize }},
$$

where SegNodesListSize is the size of the list of numbers of child nodes in segments, and SegPtrListSize is the size of the list of segment pointers. Without pointer compression, since each of the keys is associated with a segment pointer $(M=N)$, the number of entries is as the following:

$$
\frac{\text { NodeSize }-(\text { HeaderSize }+ \text { BaseKeySize })}{\text { KeySize }+ \text { SegNodesSize + SegPtrSize }},
$$

where SegNodesSize is the size for storing the number of child nodes in a segment, and SegPtrSize is the size of a segment pointer.

The Lemmas 1, 2] and 3 show the ratios computed by dividing the numbers of entries in the internal nodes in the cache conscious trees implemented using the CC-GiST by those in the existing cache conscious trees: each for the $\mathrm{CSB}^{+}$-tree [10], the pkB-tree [2], and the CR-tree [8]. In the Lemmas, it is assumed that the header size HeaderSize is four bytes, the size of a segment pointer SegPtrSize is four bytes, and the size for storing the number of child nodes in a segment SegNodesSize is one byte. For the proofs for the Lemmas, please refer to [9].

Lemma 1. For any key size, the average ratio computed by dividing the number of entries in an internal node in the existing $\mathrm{CSB}^{+}$-tree by that in the $\mathrm{CSB}^{+}$-tree implemented using the CC-GiST is as follows:

$$
1+\frac{3}{\text { NodeSize }-9} \text {. }
$$

Lemma 2. The average ratio computed by dividing the number of entries in an internal node in the existing pkB-tree by that in the pkB-tree implemented using the CC-GiST is as follows:

$$
\left(1-\frac{2}{\text { NodeSize }-4}\right) \cdot \frac{14}{13} \text {. }
$$

Lemma 3. The average ratio computed by dividing the number of entries in an internal node in the existing $\mathrm{CR}$-tree by that in the CR-tree implemented using the CC-GiST is as follows:

$$
\left(1+\frac{2}{\text { NodeSize }-20}\right) \cdot \frac{7}{6}
$$


Figure 5 shows the ratios of the numbers of entries for each of the cache conscious trees given in Lemmas 1, 2, and 3 as we vary the node size. The node sizes in the figure are determined as the multiples of minimal cacheline size (64 bytes). As shown in the figure, the numbers of entries in the internal nodes in the cache conscious trees implemented using the CC-GiST are not increased much compared with those in the existing cache conscious trees. Especially, for the $\mathrm{CSB}^{+}$-tree and the pkB-tree implemented using the CC-GiST, the ratios are increased no more than $7 \%$ for all the node sizes. The CR-tree implemented using the CC-GiST has the relatively larger ratios than the other trees. However, if we don't manage the number of child nodes in a segment, whose size is SegNodesSize in Eq. (2), the ratio will be dropped.

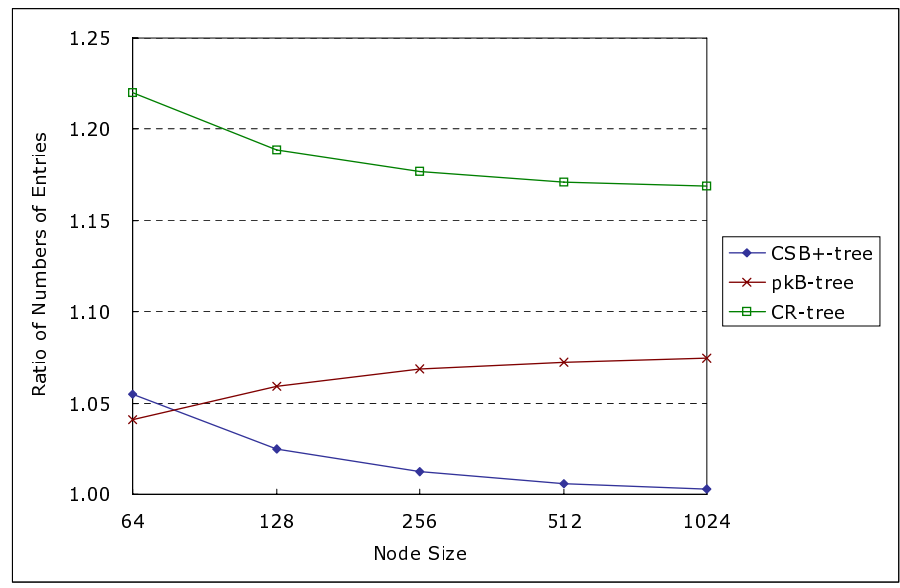

Fig. 5. Ratios of Numbers of Entries

As the result, the CC-GiST has the negligible overhead for supporting all the existing cache conscious trees in a single framework, and the performance of the tree is almost unaffected. We analyzed the ratios of the numbers of entries only for the internal nodes in this section; however, since the leaf nodes of the CCGiST have almost the same structures as those of the existing cache conscious trees, we expect to have almost the same performance for the leaf nodes.

\section{Conclusions}

In this paper, we provided the formal analysis on the performance of the CCGiST. As the result, the CC-GiST has the negligible overhead for supporting all the existing cache conscious trees in a single framework, and the performance of the tree is almost unaffected. The CC-GiST can be used not only to easily implement the existing cache conscious trees but also to develop a new tree integrating all the advantages of the existing cache conscious trees. 


\section{Acknowledgement}

This work was supported by Korea Research Foundation Grant (KRF-2003-003D00347).

\section{References}

1. A. Ailamaki, D. J. DeWitt, M. D. Hill, and D. A. Wood, "DBMS on a Modern Processor: Where Does Time Go?," In Proc. Int'l Conf. Very Large Databases, pp. 54-65, Edinburgh, Scotland, UK, Sept. 1999.

2. P. Bohannon, P. Mcilroy, and R. Rastogi, "Main-Memory Index Structures with Fixed-Size Partial Keys," In Proc. ACM SIGMOD/PODS Int'l Conf. Management of Data, pp. 163-174, Santa Barbara, California, May 2001.

3. P. A. Boncz et al., "Database Architecture Optimized for the New Bottleneck: Memory Access," In Proc. Int'l Conf. Very Large Databases, pp. 54-65, Edinburgh, Scotland, UK, Sept. 1999.

4. B. Calder, C. Krintz, S. John, and T. Austin, "Cache-Consious Data Placement," In Proc. 8th Conf. Architectural Support for Programming Languages and Operating Systems (ASPLOS), pp. 139-149, Oct. 1998.

5. T. M. Chilimbi, J. R. Larus, and M. D. Hill, Improving Pointer Based Codes through Cache-Conscious Data Placement, Technical Report, Computer Science Department, University of Wisconsin-Madison, 1998.

6. T. M. Chilimbi, J. R. Larus, and M. D. Hill, "Making Pointer Based Data Structures Cache Conscious," IEEE Computer, Vol. 33, No. 12, pp. 67-74, Dec. 2000.

7. J. M. Hellerstein, J. F. Naughton, and A. Pfeffer, "Generalized Search Trees for Database Systems," In Proc. Int'l Conf. Very Large Data Bases, pp. 562-573, Zurich, Switzerland, Sept. 1995.

8. K. Kim, S. K. Cha, and K. Kwon, "Optimizing Multidimensional Index Trees for Main Memory Access," In Proc. ACM SIGMOD/PODS Int'l Conf. Management of Data, pp. 139-150, Santa Barbara, California, May 2001.

9. W.-S. Kim, W.-K. Loh, and W.-S. Han, CC-GiST: Cache ConsciousGeneralized Search Tree, Technical Report, Department of Computer Engineering, Kyungpook National University, 2006 (Also available at http://www-db.knu.ac.kr/ ${ }^{\text {wshan/cc-gist.pdf). }}$

10. J. Rao and K. A. Ross, "Making B ${ }^{+}$-Trees Cache Conscious in Main Memory," In Proc. ACM-SIGMOD Int'l Conf. Management of Data, pp. 475-486, Dallas, Texas, May 2000. 